The International Journal of Engineering and Science (IJES)

|| Volume || 6 || Issue || 7 || Pages || PP 26-31 || 2017 ||

ISSN (e): $2319-1813 \operatorname{ISSN}(\mathrm{p}): 2319-1805$

THE IJES

\title{
Simulation of Parameters of Flow Field on Groove in Electrochemical Machining
}

\author{
*Jian-minWu ${ }^{1}$, Jin-dong Wang ${ }^{2}$ \\ College of Mechanical Engineering, Shanghai University of Engineering Science, Shanghai 201620, China \\ Corresponding Author: Jin-dong Wang
}

\begin{abstract}
Electrochemical machining (ECM) is one of the important non-traditional machining processes, which is used formachining difficult-to-cut materials and complicated shapes or special structure. In this work, taking grooves in inner cylinder for lightweightas the research objective, the simulation in certain condition was done to study the effect of parameters such as width of inlet slot, inlet pressure and machining inter-electrode gap on flow field characteristics of machining surface. Then analysis of the results was carried on, and the results showed that larger width of inlet slot was better, effect of inlet pressure was small, machining gap should be comprehensively chosen. It is helpful for selecting initial fluid parameters with Computational Fluid Dynamics (CFD)

Keywords: Electrochemical Machining (ECM); Flow Fields; Computational Fluid Dynamics (CFD); Groove
\end{abstract}

Date of Submission: 05 July 2017

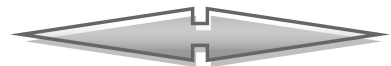

Date of Accepted: 17 July 2017

\section{INTRODUCTION}

Electrochemical machining (ECM) is based on the phenomenon of anodic dissolution, whose laws were established by Faraday in 1833 [1]. It is an advanced metal-working technique which is used to machine hard materials that are difficult or impossible to cut through traditional techniques. Due to its advantages like no tool wear, burr free, no affected layers, ease of machining complex shapes, ECM finds very wide cross-industry applications, such as aerospace and space, automotive, chemical, general engineering, maritime, etc.

However, the main disadvantages of ECM are the tool design and the selection of ECM parameters, which directly influences the machining stability and process accuracy. So recent years many scholars performed a range of experiments to explore designing the cathode and selecting the Machining parameters. Zinovi Brusilovski [2] revealedthe effect of control parameters such as operating voltage, electrolyte pressure,relative pulse duration, etc.on interelectrode gap size on the basis of experimentalstudies. C. Senthilkumar [3]et al.used a multiple regression model to represent relationship betweeninput and output variables, and applied a multi-objective optimization method based on a non-dominated sorting genetic algorithm-II(NSGA-II)to optimize ECM process.Qingming Fan [4] et al.builtthe square ECM machining gap flow field mathematical model to study the fluid distribution, and appliedCOMSOL software and computational fluid dynamics (CFD) to simulation flow field of the machining gap channels.Biswesh R. Acharya [5]et al.studied the effect of four important parameters such as current, voltage, flow rate of electrolyte and inter-electrode gap on material removal rate and surface roughness by response surface methodology, and found out the optimal process parameters with non-dominated sorted genetic algorithm. Mitsuo Uchiyama [6] et al. presented the optimization of the tool design and structure in an electrochemical curved holemachining method, and through numerical analysis ofthe electrostatic field and a large deflection to improve machiningefficiency with optimized tool. S. Ayyappan [7] et al.studied the influence of ECM process parameters such as applied voltage,inter-electrode gap and electrolyte concentration on the material removalrate and surface roughness.Liu Guodong [8]et al. researched the selection and optimization of composite electrolyte to dissolve and remove electrolyticproducts in order to machine micro holes with high aspect ratio during the process of micro electrochemical machining of metallic alloys. Dong Zhu [9]et al. studied the optimization of the distribution of flow field through the turning profile on thecathode at the electrolyte inlet to eliminate the flow marks on aero engine blades, and by optimizing the turning radius to improvethe uniformity of the distribution of flow field in the machining gap.

Although a significant amount of research has been conducted, the selection of ECM parameters and tool design still relies very much on personal knowledge and work experience. So it is necessary to carry out some further studies o these topics. In this paper, in order to optimize initial fluid parameters such as width of inlet slot, inlet pressure and machining inter-electrode gap, the simulation were carry out to investigate the effects of these parameters on low field characteristics of machining surface. 


\section{MATHEMATICAL MODEL}

Here, in ECM assumptions in inter-electrode gap are made as follows: (1)Fluid is an incompressible flow with a constant viscosity; (2)In ECM, in order to get uniform flow field and eliminate concentration polarization, the flow of electrolyte is turbulent; and (3)Dynamic equilibrium is reached.

On account of the smaller length of electrolyte flow path, so ignoring changes of temperature and energy dissipation caused by temperature for working medium during machining process, the electrolyte flow is constrained by the conservation laws for mass and momentum. The electrolyte flow in inter-electrode gap should satisfy the motion equation which are the Navier-Stokes equation for an incompressible fluid flow. The explicit form of the Navier-Stokes equation may be stated as follows[10]:

$\rho\left(\frac{\partial \mathbf{u}}{\partial t}+\mathbf{u} \cdot \nabla \mathbf{u}\right)=-\nabla p+\mu \nabla^{2} \mathbf{u}+2 \nabla \mu \cdot \mathbf{E}+\rho g$

Where, $\rho$ is density, $\mu$ is viscosity, $t$ is time, $p$ is pressure, $g$ is gravitational acceleration. And

$$
\mathbf{E}=\frac{1}{2}\left[\nabla \mathbf{u}+(\nabla \mathbf{u})^{T}\right]
$$

The four terms on the right-hand side express the pressure force, the viscous force, a force due to viscosity variations, and the body force, respectively.

In this paper, standard k-epilsion turbulence model was used to solve the problem based on the Boussinesq approximation, and it is calculated by: $[11,12]$.

$$
\begin{aligned}
& \frac{\partial}{\partial t}(\rho k)+\frac{\partial}{\partial x_{i}}\left(\rho k u_{i}\right)=\frac{\partial}{\partial x_{j}}\left[\left(\mu+\frac{\mu_{\mathrm{t}}}{\sigma_{k}}\right) \frac{\partial k}{\partial x_{j}}\right] \\
& \frac{\partial}{\partial t}(\rho \varepsilon)+\frac{\partial}{\partial x_{i}}\left(\rho u_{i}\right)=\frac{\partial}{\partial x_{j}}\left[\left(\mu+\frac{\mu_{\mathrm{t}}}{\sigma_{\varepsilon}}\right) \frac{\partial \varepsilon}{\partial x_{j}}\right]
\end{aligned}
$$$$
+\mathrm{G}_{k}-\rho \varepsilon(3)
$$

$$
+C_{1 \varepsilon} \frac{\varepsilon}{k} G_{k}-C_{2 \varepsilon} \rho \frac{\varepsilon^{2}}{k}(4)
$$

Where, $\mathrm{k}$ is the turbulent kinetic energy, $\varepsilon$ is the turbulent dissipation rate, $\mu_{\mathrm{t}}$ is the eddy viscosity, $\mathrm{G}_{\mathrm{k}}$ is the generation of turbulent kinetic energy due to the mean velocity gradients, $\sigma \mathrm{k}$ and $\sigma \varepsilon$ are the turbulent Prandtl numbers for the $\mathrm{k}$ and $\varepsilon$, respectively. $\mathrm{C}_{1 \varepsilon}$ and $\mathrm{C}_{2 \varepsilon}$ are model constants. Tubulent eddy viscosity, $\mu \mathrm{t}$, is modelled according to the Eq. 5, by combining the values of $\mathrm{k}$ and $\varepsilon$.

$$
\mu_{\mathrm{t}}=\rho \mathrm{C}_{\mu} \frac{k^{2}}{\varepsilon}
$$

The equations contain five dimensionless constants: $\mathrm{C}_{1 \varepsilon}, \mathrm{C}_{2 \varepsilon}, \mathrm{C}_{\mu}, \sigma_{\mathrm{k}}, \sigma_{\varepsilon}$. The standard k- $\varepsilon$ model employs following values for the constants [13]:

$$
\mathrm{C}_{1 \varepsilon}=1.44, \mathrm{C}_{2 \varepsilon}=1.92, \mathrm{C}_{\mu}=0.09, \sigma_{\mathrm{k}}=1.0, \sigma_{\varepsilon}=1.3
$$

\section{RESULTS AND DISCUSSIONS}

In ECM, the fist step is to design the tool, then determining the machining parameters such as inlet pressure and machining gap. So in this paper, the effect of width of inlet slot was first considered, after that the simulation of effect of inlet pressure and machining gap were performed.

\subsection{The simulation of width of inlet slot}

Here three width were considered, that is ws $=1.2 \mathrm{~mm}$, ws $=1.5 \mathrm{~mm}$, ws $=1.8 \mathrm{~mm}$, and inlet pressure $\mathrm{P} 0=0.6 \mathrm{MPa}$, inter-electrode gap $\Delta=0.5 \mathrm{~mm}$. The results were as follows.
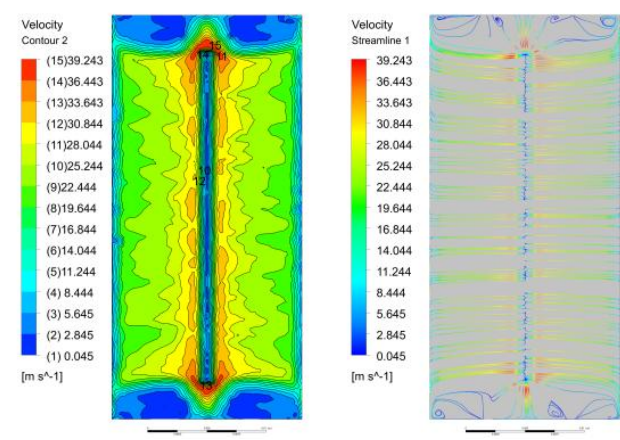

(a) Width of inlet slot ws $=1.2 \mathrm{~mm}$ 

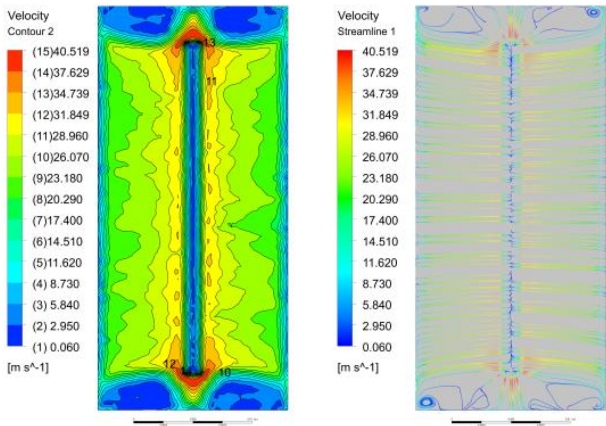

(b) Width of inlet slot ws $=1.5 \mathrm{~mm}$
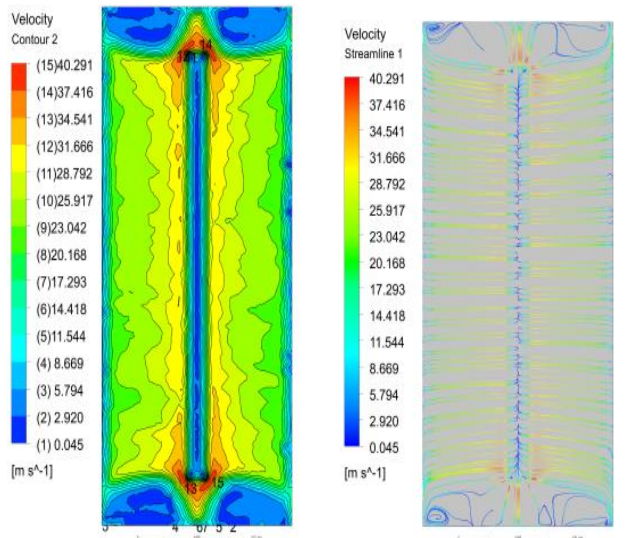

(c) Width of inlet slot ws $=\overline{1.8} \mathrm{~mm}$

Fig.1 Velocity Contour and streamline of width of inlet slot ws=1.2, 1.5,1.8 mm, respectively

From the fig.1, we can observed that the regions with smaller velocity (such as less than $3 \mathrm{~m} / \mathrm{s}$ ) were mainly located in both sides of the width of the rectangular. And with the increase of the width of inlet slot, the total areas with smaller velocity (such as $3 \mathrm{~m} / \mathrm{s}$ ) appeared to be waning although the variations were not obvious. From the velocity streamline, streamlines at the both ends were sparse which illustrated that these regions were not main stream, and had smaller electrolyte flow rate. And smaller flow rate probably could not timely carry away the corrosion products. But it is important to note that with the width of the inlet slot increases, raising the possibility of the presence of the convex on the machined surface. So for the width of slot, it should not be automatically assumed that wider is better.

\subsection{The simulation of inlet pressure}

Here three inlet pressures were considered, that is $\mathrm{P} 0=0.4 \mathrm{MPa}, \mathrm{P} 0=0.6 \mathrm{MPa}, \mathrm{P} 0=0.8 \mathrm{MPa}$, and width of inlet slot is ws $=1.5 \mathrm{~mm}$, inter-electrode gap $\Delta=0.5 \mathrm{~mm}$. The results were as follows.
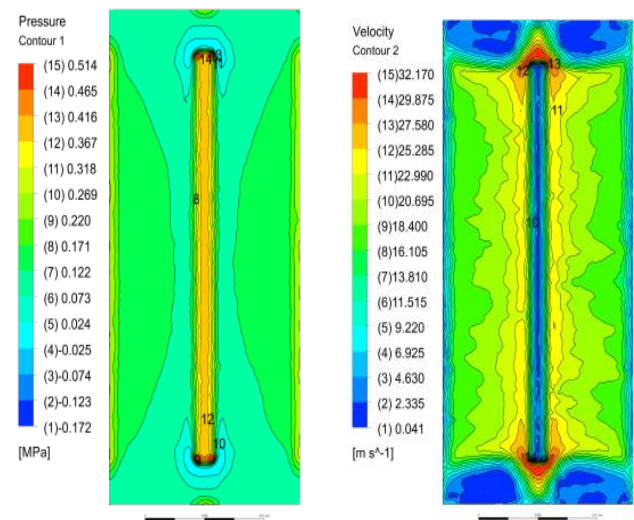

(a) Inlet pressure $\mathrm{P} 0=0.4 \overline{\mathrm{MPa}}$ 

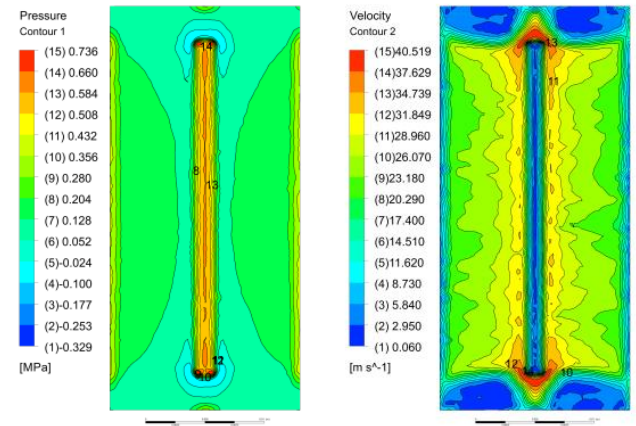

(b) Inlet pressure $\mathrm{P} 0=0.6 \mathrm{MPa}$
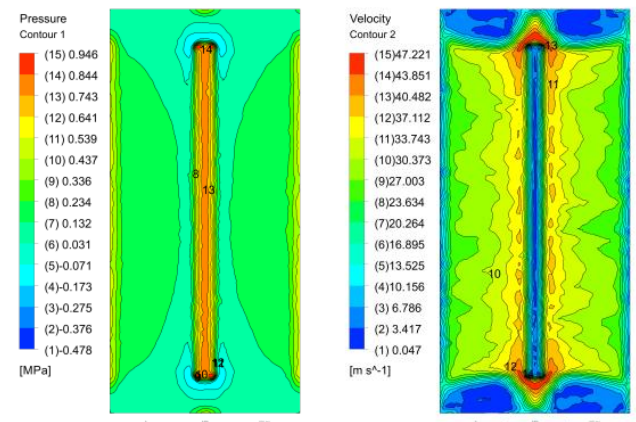

(c) Inlet pressure $\mathrm{P} 0=0.8 \mathrm{MPa}$

Fig.2 Velocity Contour and streamline of inlet pressure $\mathrm{P} 0=0.4,0.6,0.8 \mathrm{MPa}$, respectively

In ECM, sufficient electrolyte must be supplied in the inter-electrode gap in order to carry away the electrolytic products in time. But usually it is difficult to measure the flow rate and velocity of electrolyte in machining regions, so inlet pressure is applied to guarantee the fluid parameters. From pressure contours (Fig.2), we could find that the pressure on the machining surface increased and was higher than inlet pressure, and the rate of increase declined gradually with the rise of inlet pressure. It can also be seen that there were no significant differences in distributions of pressure among these three inlet pressures under the condition that the flowing electrolyte holds the turbulent state.

As can be seen from velocity contour shown in Fig., the rate of change in electrolyte velocity raised with the augmentation of the inlet pressure, however, it is not beneficial to electrochemical machining with higher rate of change in electrolyte velocity.

\subsection{The simulation of machining gap}

Here three machining gap were considered, that is $\Delta=0.3 \mathrm{~mm}, \Delta=0.4 \mathrm{~mm}, \Delta=0.5 \mathrm{~mm}$, and width of inlet slot is $\mathrm{ws}=1.5 \mathrm{~mm}$, inlet pressure $\mathrm{P} 0=0.6 \mathrm{MPa}$. The results were as follows.
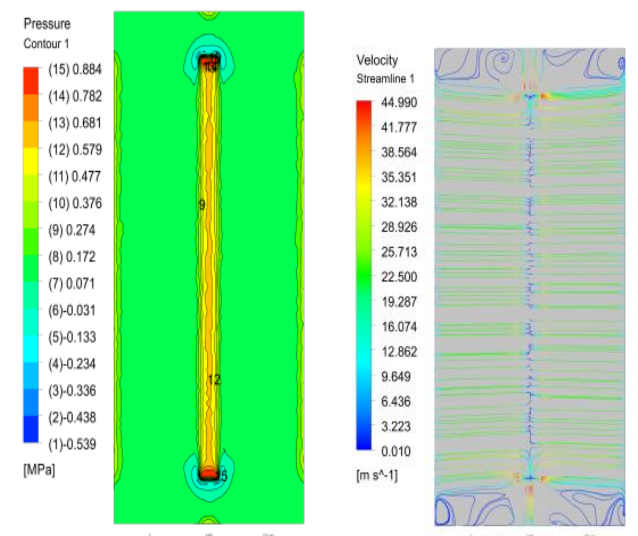

(a) Inter-electrode gap $\Delta=0.3 \mathrm{~mm}$ 

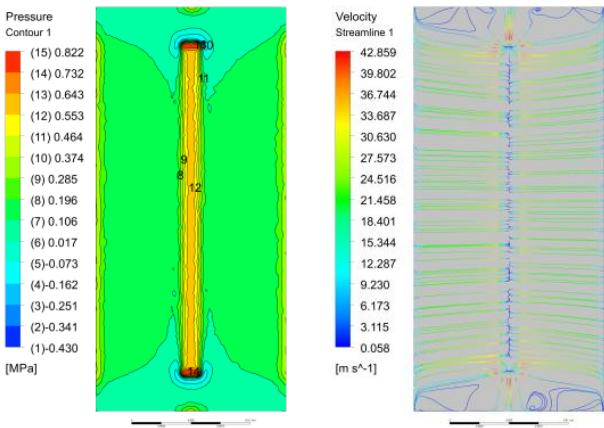

(b) Inter-electrode gap $\Delta=0.4 \mathrm{~mm}$
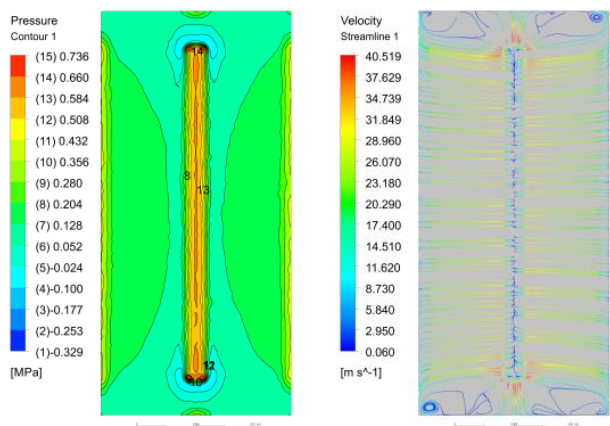

(c) Inter-electrode gap $\Delta=0.5 \mathrm{~mm}$

Fig.3 Velocity Contour and streamline of inter-electrode gap $\Delta=0.3,0.4,0.5 \mathrm{~mm}$, respectively

From pressure contour (Fig.3), it can be seen that with the decrease of the gap, the pressure in the gap increased. Furthermore, the more smaller inter-electrode gap was, the more higher the pressure grew, the more uniform the pressure distribution became. Uniformity of pressure means consistent or even of electrolyte velocity, which is beneficial to ECM.

Along the length direction, the flow lines on both sides of the slot were more evenly distributed, and the streamlines were sparse at both ends of width which illustrated that the flow rate of electrolyte was smaller compared to other parts of the whole machining surface. Furthermore, vortices appeared at both ends of width, and the vortices weakened as the gap increased. The presence of vortex showed that the flow field was in disorder, and the electrolyte velocity was influenced and became smaller in these regions.

Under the condition of same slot width and inlet pressure, smaller inter-electrode gap was conducive to machining on the basis of the pressure. But bigger inter-electrode gap was helpful to machining based on streamline contour. These two results were conflicting to each other. So the determination of inter-electrode gap should take into account other machining parameters.

\section{EXPERIMENTS}

Some experiments were carried out to testify the results of simulation. In experiment, the width of inlet slot was ws $=1.5 \mathrm{~mm}$, inlet pressure was $\mathrm{P} 0=0.5 \mathrm{Mpa}$, the electrolytewas $\mathrm{NaNO}_{3}(20 \%$ mass fraction), and its temperature was controlled at $28 \pm 1^{\circ} \mathrm{C}$.

Fig.4 shows the machined test piece. It can be seen that there were some convexity and over-cut on the machined surface at the both ends. This was coincide with simulation results which there were smaller electrolyte flow at the both ends. This defects should be eliminated by combination of tool structure and appropriate machining parameters.

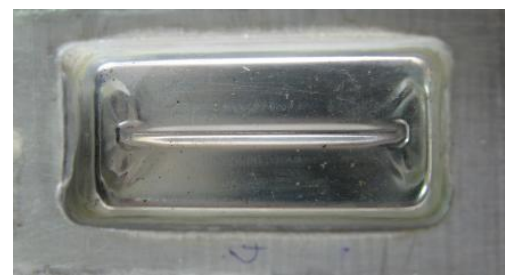

Fig. 4 Picture of machined groove 


\section{CONCLUSIONS}

After simulations and some experiments, some conclusions from this paper can be summarized as follows:

- Simulation results illustrated that change of inlet pressure had little effect on flow field and could be neglected based on the premise that electrolyte flow is in turbulent state.

- Experimental results were consistent with simulation results.

- It would be feasible that some fluid parameters were initially chosen with the help of Computational Fluid Dynamics (CFD).

\section{REFERENCES}

[1]. R. Venkata Rao. Advanced Modeling and Optimization of Manufacturing Processes: International Research and Development. Springer-Verlag London Limited. 2011.

[2]. Zinovi Brusilovski. Adjustment and readjustment of electrochemical machinesand control of the process parameters in machiningshaped surfaces. Journal of materials processing technology 196(2008): 311-320

[3]. C. SENTHILKUMAR, G. GANESAN, R. KARTHIKEYAN. Parametric optimization of electrochemical machining ofAl/15\% SiCp composites using NSGA-II. Trans. Nonferrous Met. Soc. China 21(2011):2294-2300.

[4]. Qingming Fan, Geng Liu, Zhijian Fan, Yaqi Hu. Flow Field Numerical Simulation of the ECM Machining Gap on Square Holes Based on COMSOL.Published in: Circuits, Communications and System (PACCS), 2011 Third Pacific-Asia Conference on Wuhan, China.

[5]. Biswesh R. Acharya, Chinmaya P. Mohanty, S.S. Mahapatra. Multi-objective Optimization of Electrochemical Machining of Hardened Steel Using NSGAII. Procedia Engineering, 51(2013): 554-560.

[6]. Mitsuo Uchiyama, Masanori Kunieda. Application of large deflection analysis for tool design optimization in anelectrochemical curved hole machining method. Precision Engineering. 37 (2013): 765-770.

[7]. S. Ayyappan, K. Sivakumar. Enhancing the performance of electrochemical machiningof $20 \mathrm{MnCr} 5$ alloy steel and optimization of process parametersby PSO-DF optimizer. Int J Adv Manuf Technol (2016) 82:2053-2064

[8]. Liu Guodong, Li Yong, Kong Quancun, Tong Hao. Selection and Optimization of Electrolyte for Micro ElectrochemicalMachining on Stainless Steel 304. 18th CIRP Conference on Electro Physical and Chemical Machining (ISEM XVIII). Procedia CIRP 42 ( 2016 ) 412-417.

[9]. Dong Zhu, Ronghui Zhang, and Cheng Liu. Flow Field Improvement by Optimizing Turning Profile atElectrolyte Inlet in Electrochemical Machining. INTERNATIONAL JOURNAL OF PRECISION ENGINEERING AND MANUFACTURING, 2017, Vol. 18, No. 1, pp. 15-22

[10]. .C. Pozrikidis. Introduction to Theoretical and Computational Fluid Dynamics (Second Edition). Oxford University Press. 2011.

[11]. Chaoqun Liu. Advances in Modeling of Fluid Dynamics. Published by InTech. First published October, 2012, Printed in Croatia.

[12]. ANSYS, 2009, ANSYS FLUENT Theory Guide, ANSYS Inc, Canonsburg, PA, USA.

[13]. H K Versteeg and W Malalasekera. An Introduction to Computational Fluid Dynamics: THE FINITE VOLUME METHOD. (Second Edition). Pearson Education Limited. 2007. 